\title{
Wickedpedia: The 'iEvo’ Effect in Contemporary Education
}

\author{
J. R. Stone $\mathrm{e}^{1,2,3 \text {, * }}$ \\ ${ }^{1}$ Department of Biology, McMaster University, Hamilton, Canada \\ ${ }^{2}$ SHARCNet, McMaster University, Hamilton, Canada \\ ${ }^{3}$ The Origins Institute, McMaster University, Hamilton, Canada
}

Email address:

jstoner@mcmaster.ca

\section{To cite this article:}

J. R. Stone. Wickedpedia: The ‘iEvo’ Effect in Contemporary Education. Education Journal. Vol. 4, No. 5, 2015, pp. $259-262$. doi: $10.11648 /$ j.edu. 20150405.21

\begin{abstract}
Two hundred and seventy one students in a third year undergraduate evolution course were presented with an assignment about a fictitious theory for which information previously had been made accessible online. Among the students, approximately $90 \%$ accessed the online information, with more than half failing to demonstrate any appreciation for deception or error. This phenomenon, wherein students electronically access resources and acquire data without criticism or integration, is christened the iEvo effect. Testing after assignment feedback had been provided revealed increased appreciation for the material and Internet use, suggesting that the iEvo effect can be utilised as an online teaching practice.
\end{abstract}

Keywords: Evolution, Internet, Learning, Online, Teaching, Wikipedia

\section{Introduction}

The Internet and its increasing availability over the past quarter century have revolutionized education, imparting positive and negative changes. The most-negative effects on the learning process, ironically, may manifest in some students who are or become less lazy. With so much information accessible literally at their fingertips, most students readily acquire material in voluminous quantities. These students have mastered their information collecting skills and become proficient at presenting material in written or spoken form, often in response to modern evaluation practices $[1,2]$. But, perhaps as partial compensation for effort invested in research, some students rarely reveal any selectivity or aptitude for synthesis. I dramatically exposed this emerging trend in an undergraduate course in evolution, and the events and practices that I recount and describe herein may be adapted as a means for utilising the Internet effectively as a teaching tool.

\section{Methods}

Frustrated with students' abilities to review, record, and regurgitate information without synthesizing it, I devised a report assignment to demonstrate to them the dangers inherent in amassing and redistributing facts uncritically. I created a fictitious theory about human evolution to function as the subject for the report and, simultaneously, comment on student inabilities to integrate ideas in a discriminating manner. I christened the theory 'iEvo,' paying homage to the bourgeoning market for 'i'-products (e.g., iMac, iTunes, iPod, iPhone, iPad, iWatch), for which students have an unbridled affinity. The theory, itself, existed and would be described only at a Wikipedia page, available for students (and other, incidental Internet surfers) to discover.

In 2007 late summer, I composed text for the theory. I secured an account at Wikipedia and uploaded the text to create an iEvo page. I initiated a campaign to Google-search the terms 'iEvo' and 'Wikipedia' repeatedly, every day, for approximately one month. By September, the iEvo Wikipedia page was accessible among the top 10 hits from a Google search on only the term 'iEvo.'

iEvo was described as stating boldly that modern innovations like the Internet had halted human evolution. The theory was purported to have been founded on the basis of data demonstrating that many contemporary conveniences created by humans counteract factors that normally would impose selection on individuals in populations (the contents for the original Wikipedia page are reproduced in Appendix 1).

The population involved in the experiment comprised $n=$ 271 undergraduate students in a third-year evolution course that served as a prerequisite for two fourth-year courses (population genetics and human genetics, $\mathrm{n}=37$ and 52, respectively) and an optional prerequisite for two others. 
Most students, therefore, may be considered to have elected to have taken the course, and, consequently, the sample may be considered as representative for students in undergraduate science programs at reputable international universities.

I assigned the report on the first day in term. I presented instructions for the report electronically (i.e., using an LCD projector) as soon as students had entered the lecture hall for the first time, so effects from lecturing style and expectations on the data that ultimately would be obtained by students were minimized. The instructions read: "using as few words as you can (and no more than 250), please provide a critical report about iEvo." I provided no other information. I told the 5 teaching assistants nothing about the report in advance, providing them only with the same instructions that I had provided to the students during lecture and reiterated electronically, via the e-learning tool WebCT (BlackBoard, Inc., Washington DC).

Text at the Wikipedia page contained hints that the theory was phony. The theory was professed to have been authored by Connie Furmzorçis (i.e., 'confirm your sources') in a nonexistent journal ('e-Evolution'). The text contained a technical inaccuracy: the paper in which the theory was published was described as comprising 7 pages, but 218 pages (16-234) were listed in the citation to the corresponding, lone entry that was included in the reference section. The text also presented a fundamental conceptual inaccuracy that was intrinsic to the course subject matter (i.e., evolution) and independent from the report: an unqualified statement that selection leads to 'improvement.' I had covered this common misrepresentation in lectures during the first week in term (i.e., that all statements about selection and fitness must be qualified by reference to environment), providing sufficient time for students to have identified the error before submitting their reports.

\section{Results and Discussion}

The overwhelming majority among the students $-89 \%$ on the basis of teaching assistant estimates - uncritically 'copyand-paste'd the information from the iEvo Wikipedia page into files, usually with slight textual emendation, to produce their reports. As evolution technically is among the mostaccessible major scientific theories, this particular phenomenon may be interpreted as resulting from typical, habitual learning behavior for undergraduate students in science programs, at least in reputable North American universities. Evidence that some students had invested more effort was detected when the course instructor was contacted via voicemail by library staff and visited in person by the Sciences Liaison Librarian. These librarians had been consulted by the more-invested students and were as puzzled as were their clients about the report. The Sciences Liaison Librarian was informed about the experiment, who, appreciating the objective, summarily informed the library staff.

Less than half - approximately $42 \%$ - among the students who accessed the Wikipedia page noticed that the theory was a hoax or, at least, suspicious (Figure 1); approximately $20 \%$ reproduced the information from the Wikipedia page but with commentary; and approximately $2 \%$ mentioned that they were unable to obtain the lone reference (electronic or hardcopy). Only approximately $11 \%$ noticed the 'planted' technical or conceptual inaccuracies.

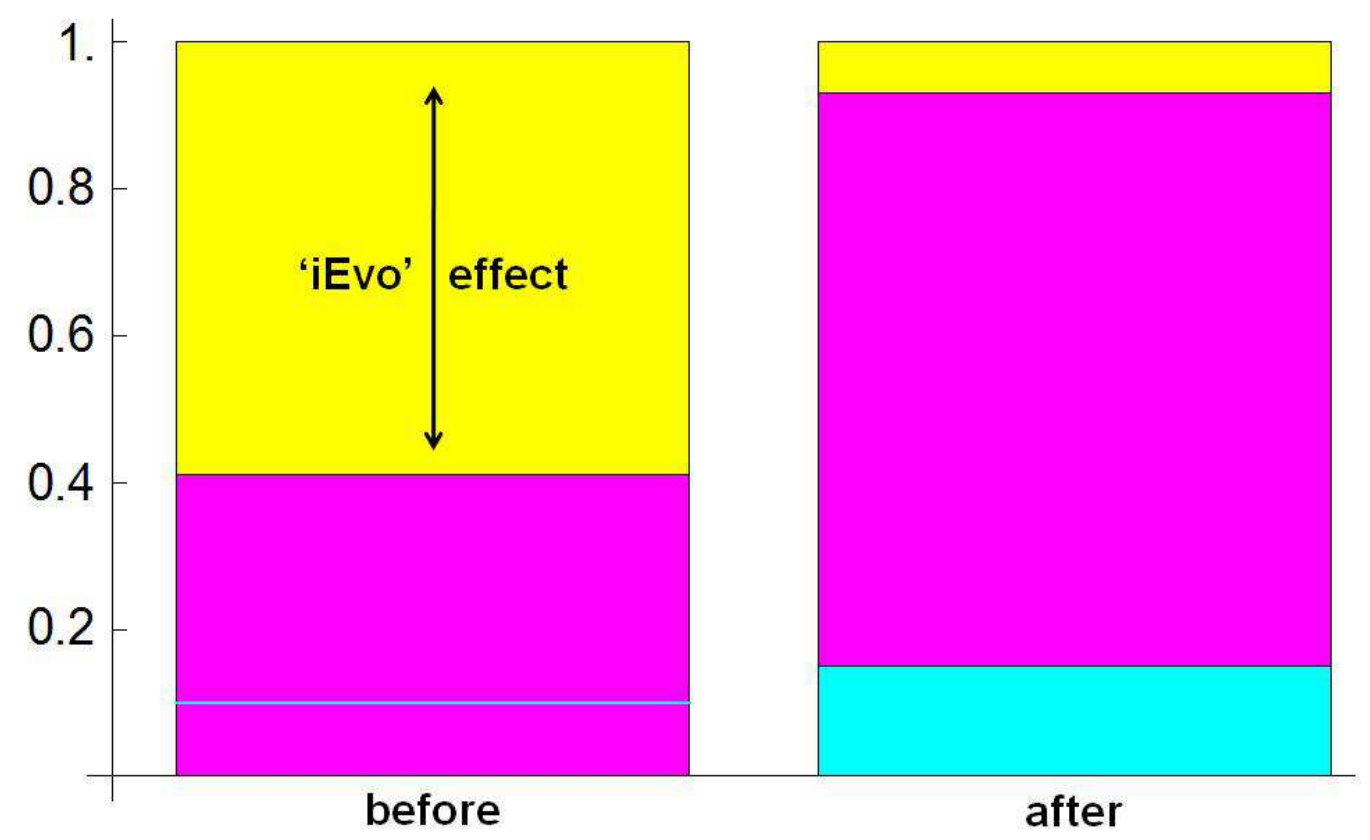

Figure 1. Proportionate response-types by students. The bar at the left represents responses to an assignment posed at term initiation, in which most students transferred information from a Wikipedia page about a phony scientific theory (iEvo) for a report, without (yellow) or with (magenta) assessing the information; the thin line (cyan) indicates that few among the students who assessed the information (11\%) noticed technical or conceptual inaccuracies with the text, itself. The bar at the right represents responses to a related multiple choice problem posed at term end (approximately two months later), in which students demonstrated no (yellow), partial (magenta), or complete (cyan) learning to have taken place. 
One bright student, who probably realized the scam and its purpose, revised the text at the Wikipedia page. This hopefully emphasized the intent to other students. To test whether that intent had been appreciated, I included in the 'open-book' (and open-notes) examination to end the course, approximately two months later, one related multiple choice problem (which received post hoc a difficulty index $\mathrm{P}(\mathrm{diff})=$ 0.153; that problem is reproduced in Appendix 2). Results generated by students were encouraging, albeit modestly. Approximately $15 \%$ responded to the problem perfectly; approximately $78 \%$ demonstrated at least partial appreciation for the material and Internet use; and approximately 7\% still missed the point completely.

\section{Conclusion}

Students actively amassed information from the Internet but mostly in an undiscerning and disintegrative manner; the pattern associated with this behavior is dubbed the iEvo effect (Figure 1, left). The iEvo effect may be utilised as an effective educational tool and overcome (Figure 1, right).

Online resources, like the Internet, itself, constitute fascinating and innovative human achievements. The pages at Wikipedia, like the one concocted for the experiment described herein, generally contain accurate information, though sometimes (temporarily) lacking in breadth and depth, and constitute a valuable repository for student learning in many forms [3-5]. Until a consensus is reached on how Internet resources like Wikipedia can be utilised for learning, educators should counsel students to interpret information that is accessible at those pages with caution, and consider Wikipedia, itself, as a portal, providing first-access to general information and sources [6]. Educators ought to adopt moreactive roles and consider Internet resources like Wikipedia as exciting teaching tools [7], with which teachers can promote research-based composition for online contribution as invaluable means for facilitating local and global learning [8] and avoiding the 'iEvo' effect (Figure 1) $[9,10]$.

Appendix 1: Supporting Text-iEvo Wikipedia Page

The term 'iEvo' is the shortened form for 'iEvolution,' the name given to the theory that modern innovations like the internet have halted human evolution. The theory was proposed by Connie Furmzorçis.

The theory involves the notion that the human lineage has stopped evolving because many contemporary conveniences created by humans are counteracting the factors that normally would impose selection regimes that would lead to improvements. For instance, glasses enable humans with imperfect vision to perform tasks as effectively as do humans with perfect vision (all other things being equal) and, so, can achieve equivalent fitness.

The theory was developed on the basis of empirical data obtained from experiments in which undergraduate students who use the internet in a cautious, complementary manner, the 'cc' group, were compared to undergraduate students who use the internet in an extensive and exclusive manner, the 'ee' group. The students were assessed on the basis of their abilities to intake information, abstract and assimilate concepts, and apply them to solve problems. Students in the cc group outperformed their peers in understanding subjects and assimilating ideas with their worldviews (a long-term, fitness measure), while students in the ee group outperformed their peers in delivering detailed presentations and reports (a short-term, performance measure).

The 7-page theory was published in the electronic journal 'e-Evolution.'

References

Con. Furmzorçis. 2007. iEvo: a new perspective on human evolution. e-Evolution 17:16-234.

Appendix 2: Supporting Text - iEvo Multiple Choice Problem

Please select any and all responses that complete accurately the following statement.

An inaccuracy that is associated with iEvo as it was presented at the Wikipedia page involved

A. its description as a 7-page theory and the page numbers that were cited in the journal 'e-Evolution.'

B. its claim that human evolution has been slowed by modern innovations like the Internet.

C. its claim that glasses effectively confer unto humans with imperfect eyesight vision equivalent to humans with perfect eyesight.

D. the sample sizes that were used in the experiment that is described at the page, which were too small.

E. its implication that modern innovations could affect human evolution.

Appendix 3: Supporting Text-Fate for the iEvo Wikipedia Page

A search at the Internet site Wikipedia (http://www.wikipedia.org) for information about the term 'iEvo' currently returns 0 matches. In 2008 (i.e., soon after the course had ended), a search returned 4 links from 2007, among which one listed entries from an online discussion, held on December 06 through 08, about suspicions that iEvo might have constituted a "hoax;" another listed iEvo as an article (number 36) that had been scheduled for deletion on December 06; yet another explained that a page containing information about iEvo had been 'reverted' as suspected vandalism; and a final one confirmed the aforementioned deletion.

\section{Acknowledgments}

T. Berners-Lee provided resources and Herky-Tiffany Stone and L. A. Kings provided enthusiasm to run the experiment.

\section{References}

[1] B. Alberts, "Redefining science education," Science vol. 323, p. $437,2009$. 
[2] H. Arsham, "Impact of the Internet on learning and teaching," USDLA Journal vol. 16, issue 3, 2002.

[3] A. Forte, A. Bruckman, "From Wikipedia to the classroom: exploring online publication and learning," in Proceedings of the 7th International Conference of the Learning Sciences, S. A. Barab, K. E. Hay, D. T. Hickey, Eds. Mawah: International Society of the Learning Sciences, 2006, pp.182-188.

[4] D. Murley, "In defense of Wikipedia," Law Lib. J. vol. 100, pp. 593-599, 2008.

[5] N. J. Schweitzer, "Wikipedia and psychology: coverage of concepts and its use by undergraduate students", Teach. Psych., vol. 35, pp. 81-85, 2008.

[6] M. Tekerek, O. Ercan, "Analysis of teachers' attitudes towards Internet use: example of chemistry teachers," Creative Ed. vol. 3, pp. 296-303, 2012.
[7] C. E. Wieman, K. K. Perkins, "A powerful tool for teaching science,” Nature Phys., vol. 2, pp. 290-292, 2006.

[8] P. Wolcott, S. E. Goodman, "Global diffusion of the Internet - I: India: is the elephant learning to dance?" Communications of the Association for Information Systems: Vol. 11, Article 32.

[9] Xeric-humor led to the term 'iEvo effect' being coined in a conversation between the author and R. K. Logan, during a lecture in the course ORIGINS 3F03 Origins of Humanity.

[10] Zestful public reaction to and the fate for the iEvo page are described in Appendix 3; subsequent to the events described herein, the name 'Ievo' was adopted by an unrelated Internet company in Canada and later 'ievo' was adopted by a biometrics company in England. 